\title{
Antiviral activity of Bifidobacterium adolescentis SPM1005-A on human papillomavirus type 16
}

Min-Kyeong Cha', Do-Kyung Lee ${ }^{1}$, Hyang-Mi An¹, Si-Won Lee', Seon-Hee Shin², Jeong-Hyun Kwon ${ }^{3}$, Kyung-Jae $\mathrm{Kim}^{1}$ and Nam-JoO $\mathrm{Ha}^{1 *}$

\begin{abstract}
Background: Probiotic lactic acid bacteria (LAB) support a functional and balanced immune system, and contribute to immune modulatory effects in combatting microbial pathogens, including viruses. Most cervical cancers are associated with anogenital region infection with high-risk (HR) human papillomavirus (HPV). In this study, we analyzed the antiviral activity of Bifidobacterium adolescentis SPM1005-A in the SiHa cervical cancer cell line expressing HPV type 16.

Methods: We assessed the cellular toxicity of B. adolescentis SPM1005-A in SiHa cells by the Trypan blue dye exclusion assay. Cells $\left(3.6 \times 10^{5}\right)$ in culture plates with or without B. adolescentis SPM1005-A in the same type of medium, were incubated with HPV type 16 at a concentration of $5.1 \times 10^{7} \mathrm{cfu} / \mathrm{ml}$. For antiviral analysis, we performed quantitative real-time PCR (qRT-PCR) for E6 and E7 oncogene expressions and observed protein levels by immunoblotting.
\end{abstract}

Results: The qRT-PCR results showed that E6 and E7 mRNA levels decreased simultaneously. Western blot analysis revealed that the E6 protein expression slightly decreased after 24 and $48 \mathrm{~h}$, but the level of E7 protein expression appear unaffected compared with that in the control. Decreased HPV16 E6 and E7 mRNA transcript and protein levels were not associated with cell morphology or significant cytotoxic effects.

Conclusions: This study showed that B. adolescentis SPM1005-A had antiviral activity through suppression E6 and E7 oncogene expression. The results suggest that B. adolescentis SPM1005-A could be potential applications of HPV-associated cervical cancer prevention.

Keywords: antiviral activity, Bifidobacterium adolescentis SPM1005-A, human papillomavirus (HPV) type 16, quantitative real-time PCR (qRT-PCR)

\section{Background}

Cervical cancer is the second most common malignant disease of the female reproductive organs, with an incidence per year of almost half a million and a mortality rate of approximately $25 \%$ [1]. Most cervical cancers are associated with the anogenital region or mucosa cell infection with human papillomavirus (HPV) [2]. Of the more than 200 different HPV types identified, $30 \mathrm{HPV}$ types infect the anogenital skin and oral mucosa and can be further classified as low risk (LR) or high risk (HR) based on the clinical prognosis of their associated lesions [3]. Approximately $99.7 \%$ of cervical cancers contain viral

\footnotetext{
*Correspondence: hanj@syu.ac.kr

${ }^{1}$ College of Pharmacy, Sahmyook University, Seoul, Republic of Korea Full list of author information is available at the end of the article
}

DNA of HR types, with type 16 being the most prevalent, followed by types $18,31,33$ and 45 [4]. The malignant phenotype of HR types depends on the expression of two viral genes E6 and E7, which bind to p53 and retinoblastoma protein $(\mathrm{pRb})$ and neutralize their function, respectively [5]. The most important function of E6 protein is binding of the tumor suppressor p53, which leads to it degradation through an ubiquitin proteolytic pathway. Degradation of $\mathrm{p} 53$ bypasses the normal growth arrest signals at the G1/S and G2/M checkpoints and is the major cause of chromosomal instability, with mutational consequences for HPV-positive cells [6]. The E7 protein interacts with $\mathrm{pRb}$ and releases transcription factor E2F, which induces expression of genes involved in cellular differentiation and proliferation $[7,8]$. Therefore, the
C Biomed Central 
studies for inhibitors of the oncogenic proteins E6 and E7 of HPV type 16 are constantly in progress.

Lactic acid bacteria (LAB) are widely used and generally recognized as safe organisms for animal and human applications. They produce antimicrobial substances such as organic acids, hydrogen peroxide, diacetyl and bacteriocins, which have beneficial effects on the host organisms [9]. Probiotic LAB support functional and balanced immune systems and contribute to immune modulatory effects in combatting microbial pathogens, including viruses [10]. Several studies have reported that LAB such as Lactobacilli boost the antiviral effect against human rotaviruses that cause diarrhea, human immunodeficiency virus type 1 and influenza virus [11-13].

Among commensal bacteria, Bifidobacteria is one of the most numerous probiotics in the mammalian gut that belong to LAB [14]. Xiao et al. have reported cholesterol reduction by a supplement containing Bifidobacterium longum, and Le Leu et al. reported the potential of Bifidobacterium animalis subspecies Lactis to prevent colorectal cancer. Also, antitumor activity has been studied in peptidoglycans isolated from a Bifidobacterium infantis strain [15-17]. Despite the various literatures indicating a protective effect of Bifidobacteria in epidemiological studies, the antiviral effects have not yet been studied in detail. We therefore assessed the antiviral activity of B. adolescentis SPM1005-A on E6 and E7 mRNA transcript and protein levels in the SiHa cervical cancer cell line expressing HPV type 16 in vitro.

\section{Methods}

Preparation of B. adolescentis SPM1005-A

For the isolation of Bifidobacteria, fecal samples were collected from healthy Koreans (aged 20 to 30 years old) by BD BBLanaerobic sample collection and transport system (Becton Dickinson and Co, USA) to maintain anaerobic conditions. Fecal samples were serially diluted tenfold from $10^{-1}$ to $10^{-8}$, and $100 \mu \mathrm{l}$ were spread into selective blood liver agar (Nissui Pharm, Japan) containing 5\% sheep blood. After $48 \mathrm{~h}$ of incubation in anaerobic conditions $\left(90 \% \mathrm{~N}_{2}, 5 \% \mathrm{H}_{2}, 5 \% \mathrm{CO}_{2}\right.$ ) (Bactron Anaerobic Chamber, USA) at $37^{\circ} \mathrm{C}$, brown or reddish-brown colonies $2 \mathrm{~mm}$ to $3 \mathrm{~mm}$ in diameter were selected for further identification [18]. A fructose-6-phosphate phosphoketolase (F6PPK) test was performed to ensure that the colonies selected were Bifidobacteria [19]. To identify the isolated Bifidobacterium spp. at the species level, 16S rRNA sequencing was performed by Bio leaders (Daejeon, Korea). We established an $N$-methyl- $N$ 'nitro- $N$-nitrosoguanidine (MNNG)-induced mutant of $B$. adolescentis SPM1005, which we named SPM1005-A. B. adolescentis SPM1005-A, was cultured at $37^{\circ} \mathrm{C}$ for $48 \mathrm{~h}$ on general anaerobic medium (GAM, Nissui Pharm, Japan) under anaerobic conditions and then centrifuged at 1,200 rpm for 15 minutes. The supernatant was separated from the bacterial cell pellet and filtered using an $0.2-\mu \mathrm{m}$ syringe filter (Sartorius Stedim Biotech, Germany). The purified supernatant was used for further experiments. Written informed consents were obtained from all volunteer who provided samples and the protocol was approved by the Institution Review Board of Office of Research Development, Sahmyook University.

\section{Cell culture and treatment}

SiHa cervical cancer cells expressing HPV type 16 were obtained from the American Type Culture Collection http://www.atcc.org. The cell lines were grown in Minimum essential medium alpha (Gibco) containing 10\% heat-inactivated fetal bovine serum (Sigma, USA), 10,000 $\mathrm{U} / \mathrm{ml}$ penicillin and $10,000 \mu \mathrm{g} / \mathrm{ml}$ streptomycin in a humidified incubator at $37^{\circ} \mathrm{C}$ with $5 \% \mathrm{CO}_{2}$. SiHa cells $\left(1.0 \times 10^{5}\right.$ cells per flask) were incubated for 0,24 , or $48 \mathrm{~h}$ in the presence or absence of $B$. adolescentis SPM1005-A at a concentration of $5.1 \times 10^{7} \mathrm{cfu} / \mathrm{ml}$. After incubation, the culture medium was removed and the monolayers were washed with phosphate-buffered saline (PBS) without phenol red and supplements; the cells were then immediately used for total protein and RNA extraction.

\section{Cell viability assay}

Cell viability was determined by a Trypan blue dye exclusion assay. Cells $\left(3.6 \times 10^{5}\right)$ in culture plates were incubated overnight, and then the medium was changed to new medium either with or without $B$. adolescentis SPM1005-A at a concentration of $5.1 \times 10^{7} \mathrm{cfu} / \mathrm{ml}$, and incubated for $0,24,48$, and $72 \mathrm{~h}$. After incubation, each cell suspension was mixed with an equal volume of $0.3 \%$ Trypan blue solution (Samchun Chemical, Korea). Finally, cells were observed under a microscope, and living cells were counted on hemocytometer. Morphological changes were observed using an inverted microscope (Olympus, Japan) at $\times 400$ magnification. Each assay was performed three times in triplicate and results presented as \% of control.

\section{Reverse transcription and quantitative real-time PCR (qRT-PCR)}

Total RNA was extracted from the cell line using RNeasy mini Kit (Qiagen, USA) according to the manufacturer's recommendation. RNA concentration was quantified by measuring the absorbance at $260 \mathrm{~nm}$. In order to analyze the same amount of cDNA in every sample, we performed reverse transcription as a separate step from PCR. The mRNA was reverse transcribed into cDNA using oligo-dT primer and Omniscript reverse transcription kit (Qiagen). 
The cDNA was stored at $-20^{\circ} \mathrm{C}$ or directly used in qRTPCR. As growth of HPV-transformed cervical cancer cells is dependent on sustained viral oncogene E6 and E7 expression, we investigated the inhibitory effects of B. adolescentis SPM1005-A on the cervical carcinoma cell line, $\mathrm{SiHa}$, with quantitative real-time PCR (qRT-PCR), which is an excellent method for quantitation of viral DNA $[20,21]$.

qRT-PCR was conducted in a Light Cycler (Roche) system and the data were analyzed with Light Cycler software version 4.5. The quantity of HPV16-E6 and HPV16-E7 transcripts in each sample was standardized to glyceraldehyde 3-phosphate dehydrogenase (GAPDH) transcript levels. For absolute quantification, cDNA constructed with specific binding sites for HPV16 E6 and E7 primers were used to derive the standard curves. Reactions contained $2 \mu \mathrm{l}$ each of the cDNA solution, $\mathrm{MgCl}_{2}$, primers and Fast Start DNA Master SYBR Green I (Qiagen). The primer sequences used for qRT-PCR of HPV16-E6 were 5'-GAC CCA GAA AGT TAC CAC AG-3' (nucleotide 44 to nucleotide 64) and 5'-CAT AAA TCC CGA AAA GCA AAG-3' (nucleotide 153 to nucleotide 173), and of E7 were 5'-GGA GGA GGA TGA AAT AGA TGG-3' (nucleotide 99 to nucleotide 199) and 5'-TGA GAA CAG ATG GGG CAC AC-3' (nucleotide 268 to nucleotide 287). GAPDH was used as internal control with primer sequences of 5'-CTG CAC CAC CAA CTG CTT AG-3' (forward) and 5'-TTC TGG GTG GCA GTG ATG-3' (reverse) [22]. The amplification conditions were initial incubation at $95^{\circ} \mathrm{C}$ for 10 minutes, followed by 45 cycles of $95^{\circ} \mathrm{C}$ for $10 \mathrm{~s}, 60^{\circ} \mathrm{C}$ for $10 \mathrm{~s}$ and $72^{\circ} \mathrm{C}$ for $10 \mathrm{~s}$. Results were calculated using the $\Delta \Delta \mathrm{CT}$ method with $\mathrm{cDNA}$ from all samples as a reference as described previously [23]. Positive and negative controls were included in each experiment to ensure reproducible results.

\section{Protein electrophoresis and immunoblotting}

After 24 or $48 \mathrm{~h}$ of treatment, each sample was washed with PBS and treated with lysis buffer (Thermo, USA). The samples, containing $30 \mu \mathrm{g}$ of protein, were suspended in a $2 \times$ sample buffer (Sigma, USA), boiled for 4 minutes and resolved on $15 \%$ sodium dodecyl sulfate polyacrylamide gel electrophoresis (SDS-PAGE) gels. The proteins were transferred to nitrocellulose membrane (Whatman, USA), and equal loading was verified with Ponceau S staining (Sigma). Immunodetection was performed with HPV16-E6 antibody and HPV16-E7 antibody (Santa Cruz Biotechnology, USA), followed by incubation with donkey anti-goat horseradish-peroxidase-conjugated antibody and detection using enhanced chemiluminescence western blotting detection reagents (Abfrontier). $\beta$-Actin was used as a normalization standard. Each blot is representative of three experiments.

\section{Statistical analysis}

Results were expressed as mean \pm SD. One-tailed Student's t tests were employed whenever individual data points were compared. Mean values of $P<0.05$ were considered statistically significant.

\section{Results}

\section{Effect of B. adolescentis SPM1005-A on morphology}

Representative images of the morphological changes observed after 24, 48, or $72 \mathrm{~h}$ are shown in Figure 1. Compared to control PBS-treated cells, cells exposed to B. adolescentis SPM1005-A for 72 h slowly shrank in appearance, but there was no observed difference in $24-\mathrm{h}$ to $72-\mathrm{h}$ treated cells.

\section{Cytotoxic effects}

The cytotoxicity of B. adolescentis SPM1005-A was evaluated by Trypan blue dye exclusion assay. Based on the results of the assay, the cell survival rate decreased slightly $(92,95,89 \%$, respectively) with 24 -h to 72 -h exposure to $5.1 \times 10^{7} \mathrm{cfu} / \mathrm{ml}$ B. adolescentis SPM1005A (Figure 2). This indicates that $B$. adolescentis SPM1005-A does not significantly affect the cytotoxicity of SiHa cells. Consequently, experiments to assess the antiviral activity were carried out at this tested concentration in this study.

\section{Inhibition of B. adolescentis SPM1005-A on E6 and E7 expression}

For this purpose, total RNA was converted to cDNAs for E6, E7 and GAPDH as a control by using oligo-dT primer reverse transcription. The qRT-PCR results showed a reduction of both genes mRNA transcript levels after incubating the cells with $B$. adolescentis SPM1005-A for 24 and $48 \mathrm{~h}$ (Figure 3A). PBS-treated cells were used as a control. A reduction in E6 mRNA expression was observed compared with that in untreated cells after $24 \mathrm{~h}$. In particular, E6 and E7 expression levels were significantly inhibited by $B$. adolescentis SPM1005-A treatment for $48 \mathrm{~h}\left({ }^{*} P<0.05, * * P<0.01\right)$.

\section{Western blotting}

Western blot analysis revealed that the expression of both proteins decreased slightly after incubation with $B$. adolescentis SPM1005-A (Figure 3B). Inhibition of E6 protein levels was observed at both 24 and $48 \mathrm{~h}$ in comparison to $\beta$-actin. The qRT-PCR results revealed that E7 mRNA levels decreased after treatment in the SiHa cell line, whereas the level of E7 protein expression in cells treated with $B$. adolescentis SPM1005-A appeared to be unaffected compared with that in the control. In this respect, the protein change did not consistently occur in a time-dependent manner. 

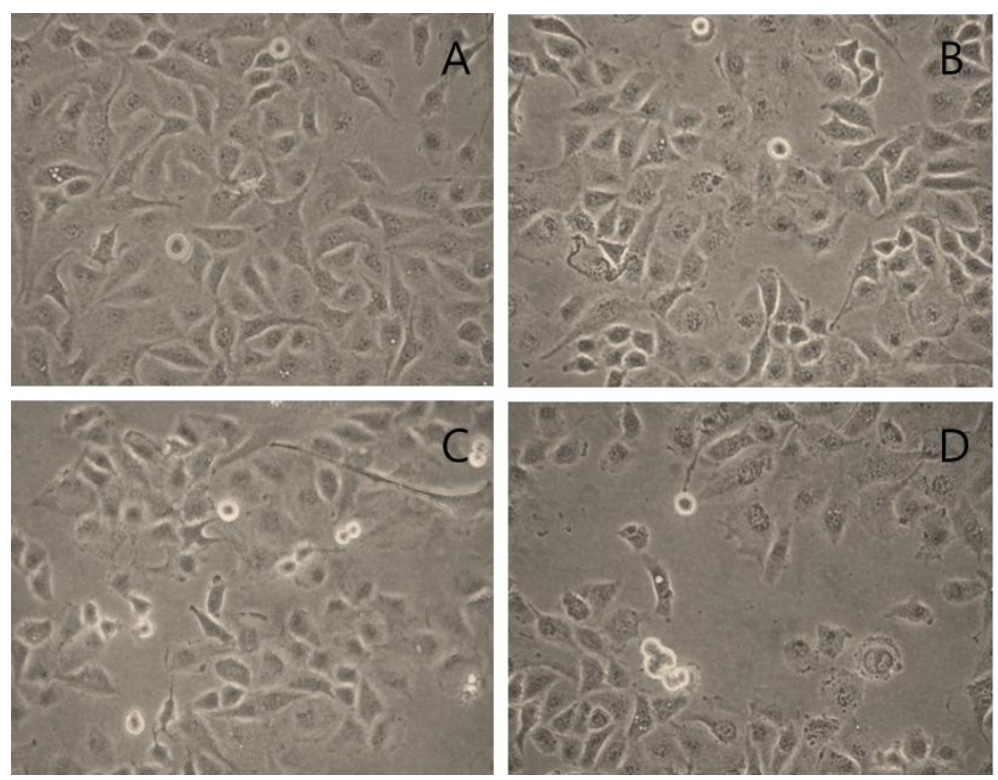

Figure 1 Morphological changes by Bifidobacterium adolescentis SPM1005-A treatment in the cervical cancer cell line SiHa. (A) Phosphate-buffered saline (PBS)-treated SiHa cells were cultured with B. adolescentis SPM1005-A at a concentration of $5.1 \times 10^{7} \mathrm{cfu} / \mathrm{ml}$ for $24 \mathrm{~h}$ (B), $48 \mathrm{~h}$ (C) and $72 \mathrm{~h}$ (D). Cells were viewed under a microscope and photographed at $\times 400$.

\section{Discussion}

Cervical cancer is the second most common malignant disease of the female and is caused by persistent infection with HPV. The majority of cases of cervical cancer develop as squamous cell carcinomas (SCCs) or adenocarcinoma [24]. The prevalence of HR HPV infection among young women is typically around $20 \%$ to $40 \%$ depending on geographical region, with the incidence lowering with age as infections are controlled by the host immune system $[25,26]$.

Probiotics, particularly Bifidobacteria, are used in fermented food and are widely assumed to be safe. Few attempts have been made recently to study the potential benefits of LAB. Lactobacillus rhamnosus GG and

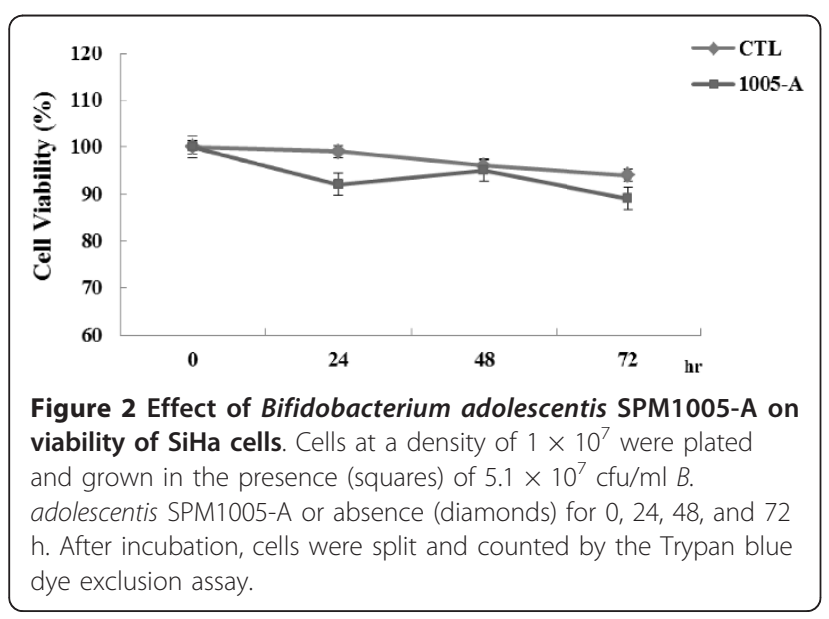

Lactobacillus acidophilus CRL431 are known to interfere with virus-induced pathology indirectly, by favoring cellular homeostasis, either stimulating innate or adaptive immunity [27]. In addition, several studies have shown that orally delivered probiotics can develop a mutually advantageous symbiosis with the gastrointestinal tract and activate immune systems by the release of proinflammatory cytokines such as tumor necrosis factor (TNF $\alpha$ ), interleukin (IL)-12, or IL-6, and by production of anti-inflammatory cytokines such as transforming growth factor $\beta$ (TGF $\beta$ ) and IL-10 [28-31]. Some Bifidobacterium species have been reported to prevent infections by pathogenic bacteria such as Escherichia coli, Salmonella and Helicobacter pylori [32-34]. Despite literature indicating the beneficial effects of Bifidobacteria, the antiviral effects have not yet been studied in cervical carcinoma cell lines. The antiviral effects of Bifidobacteria have rarely been studied in cervical cancer cell lines or in vivo, so the molecular mechanisms that underlie these effects need to be investigated in detail.

In the current study, we assessed the inhibitory effects on HPV oncogene mRNA and protein expression. As previously mentioned, the carcinogenesis process of cervical cancer is associated with the overexpression of the viral oncogenic proteins E6 and E7 that inactivate the tumor suppressors, p53 and pRb, block apoptosis, shorten telomeres and reduce immune recognition [35]. There have been several attempts to suppress these two genes typical to HR-HPV16 and HR-HPV18. Li et al. demonstrated that 


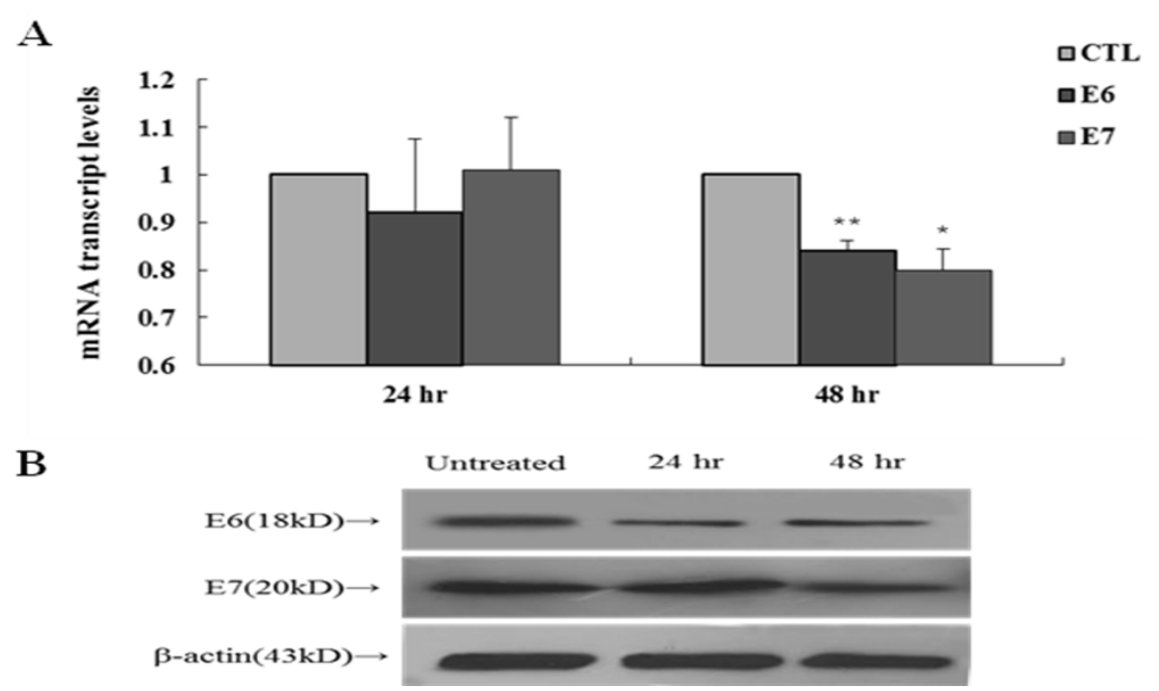

Figure 3 The expression of E6 and E7 mRNA transcript levels (A) and protein (B) in SiHa cells. SiHa cells were treated with $5.1 \times 10^{7} \mathrm{cfu} /$ $\mathrm{ml}$ Bifidobacterium adolescentis SPM1005-A for 24 or $48 \mathrm{~h}$. Transcript levels were standardized against the glyceraldehyde 3-phosphate dehydrogenase (GAPDH) mRNA level as normalization control. Each sample was evaluated by separate experiments, and results represent the means and standard deviation ( $\left.{ }^{*} P<0.05,{ }^{*} P<0.01\right)$. The cells were collected and the extracts $(30 \mu \mathrm{g})$ were resolved on $15 \%$ sodium dodecyl sulfate polyacrylamide gel electrophoresis (SDS-PAGE), transferred to nitrocellulose membranes, and immunoblotted with anti-human papillomavirus (HPV)16 E6 and anti-HPV16 E7 polyclonal antibodies. $\beta$-Actin was used as a normalization standard. The arrow indicates the location of the protein in the gel.

oncogenic gene downregulation mechanisms that increase tumor suppressor factors and induce apoptosis may be employed in cervical cancer cells [36].

Our results showed that B. adolescentis SPM1005-A can downregulate expression of both genes at both the mRNA and protein levels in SiHa cells. In particular, expression of both genes was decreased significantly with B. adolescentis SPM1005-A treatment for $48 \mathrm{~h}$. In addition, decreased HPV16 E6 and E7 gene expressions and protein levels were not associated with cell morphology and significant cytotoxic effects of $B$. adolescentis SPM1005-A in SiHa cells. However, it has been not determined how $B$. adolescentis SPM1005-A regulates the expression of E6 and E7 genes or what is the specific target region.

\section{Conclusions}

In summary, B. adolescentis SPM1005-A was found to have antiviral activity through suppression of E6 and E7 oncogene expression. The results suggest that $B$. adolescentis SPM1005-A could be useful for prevention of $\mathrm{HPV}$-associated cervical cancer.

\section{Acknowledgements}

This work (grant no. 000449270111) was supported by Business for Cooperative R\&D between Industry, Academy, and Research Institute funded Korea Small and Medium Business Administration in 2011. The authors are grateful to the College of Pharmacy of Sahmyook University and for the financial support provided by the Sahmyook University Research Fund.

\section{Author details}

${ }^{1}$ College of Pharmacy, Sahmyook University, Seoul, Republic of Korea. ${ }^{2}$ Department of Pediatrics, College of Medicine, Hallym University, Chuncheon, Republic of Korea. ${ }^{3}$ Department of Sport Medicine, Jung Won University, Chungbuk, Republic of Korea.

\section{Authors' contributions}

MKC conceived and designed the experiments. DKL, HMA, and SWL performed the experiments. SHS, JHK and KJK analyzed the data. NJH supervised the study. All authors reviewed and approved the final manuscript.

\section{Competing interests}

The authors declare that they have no competing interests.

Received: 20 April 2012 Accepted: 12 July 2012 Published: 12 July 2012

\section{References}

1. Arbyn M, Castellsagué X, de Sanjosé S, Bruni L, Saraiya M, Bray F, Ferlay J: Worldwide burden of cervical cancer in 2008. Ann Oncol 2011, 22:2675-2686

2. Campisi G, Panzarella V, Giuliani M, Lajolo C, Di Fede O, Falaschini S, Di Liberto C, Scully C, Lo Muzio L: Human papillomavirus: its identity and controversial role in oral oncogenesis, premalignant and malignant lesions. Int J Oncol 2007, 30:813-823.

3. Münger K, Baldwin A, Edwards KM, Hayakawa H, Nguyen CL, Owens M, Grace M, Huh K: Mechanisms of human papillomavirus-induced oncogenesis. J Virol 2004, 78:11451-11460.

4. Walboomers JM, Jacobs MV, Manos MM, Bosch FX, Kummer JA, Shah KV, Snijders PJ, Peto J, Meijer CJ, Muñoz N: Human papillomavirus is a necessary cause of invasive cervical cancer worldwide. J Pathol 1999, 189:12-19.

5. Um SJ, Lee SY, Kim EJ, Myoung J, Namkoong SE, Park JS: Down-regulation of human papillomavirus E6/E7 oncogene by arsenic trioxide in cervical carcinoma cells. Cancer Lett 2002, 181:11-22.

6. Thomas M, Pim D, Banks L: The role of the E6-p53 interaction in the molecular pathogenesis of HPV. Oncogene 1999, 18:7690-7700. 
7. Huh KW, DeMasi J, Ogawa H, Nakatani Y, Howley PM, Münger K: Association of the human papillomavirus type $16 \mathrm{E7}$ oncoprotein with the $600-\mathrm{kDa}$ retinoblastoma protein-associated factor, p600. Proc Natl Acad Sci USA 2005, 102:11492-11497.

8. Huh K, Zhou X, Hayakawa H, Cho JY, Libermann TA, Jin J, Harper JW, Munger K: Human papillomavirus type 16 E7 oncoprotein associated with the cullin 2 ubiquitin ligase complex, which contributes to degradation of the retinoblastoma tumor suppressor. J Virol 2007 81:9737-9747.

9. Seo BJ, Mun MR, J RK, Kim CJ, Lee I, Chang YH, Park YH: Bile tolerant Lactobacillus reuteri isolated from pig feces inhibits enteric bacterial pathogens and porcine rotavirus. Vet Res Commun 2010, 34:323-333.

10. Ivec M, Botić T, Koren S, Jakobsen M, Weingartl H, Cencic A: Interactions of macrophages with probiotic bacteria lead to increased antiviral response against vesicular stomatitis virus. Antiviral Res 2007, 75:266-274

11. Zhang W, Azevedo MS, Wen K, Gonzalez A, Saif LJ, Li G, Yousef AE, Yuan L: Probiotic Lactobacillus acidophilus enhances the immunogenicity of an oral rotavirus vaccine in gnotobiotic pigs. Vaccine 2008, 26:3655-3661.

12. Martín V, Maldonado A, Fernández L, Rodríguez JM, Connor Rl: Inhibition of human immunodeficiency virus type 1 by lactic acid bacteria from human breastmilk. Breastfeed Med 2010, 5:153-158.

13. Yasui H, Kiyoshima J, Hori T: Reduction of influenza virus titer and protection against influenza virus infection in infant mice fed Lactobacillus casei Shirota. Clin Diagn Lab Immunol 2004, 11:675-679.

14. Yin YN, Yu QF, Fu N, Liu XW, Lu FG: Effects of four Bifidobacteria on obesity in high-fat diet induced rats. World J Gastroenterol 2010, 16:3394-3401.

15. Xiao JZ, Kondo S, Takahashi N, Miyaji K, Oshida K, Hiramatsu A, Iwatsuki K, Kokubo S, Hosono A: Effects of milk products fermented by Bifidobacterium longum on blood lipids in rats and healthy adult male volunteers. J Dairy Sci 2003, 86:2452-2461.

16. Le Leu RK, Hu Y, Brown IL, Woodman RJ, Young GP: Synbiotic intervention of Bifidobacterium lactis and resistant starch protects against colorectal cancer development in rats. Carcinogenesis 2010, 31:246-251.

17. Sekine K, Ohta J, Onishi M, Tatsuki T, Shimokawa Y, Toida T, Kawashima T, Hashimoto Y: Analysis of antitumor properties of effector cells stimulated with a cell wall preparation (WPG) of Bifidobacterium infantis. Biol Pharm Bull 1995, 18:148-153.

18. Scardovi V: Genus Bifidobacterium. In Bergey's Manual of Systemic Bacteriology. Edited by: Krieg NR, Holt JG. Philadelphia, PA: Lippincott, Williams 1986:1418-1434.

19. Ahn JB: Isolation and characterization of Bifidobacterium producing exopolysaccharide. Food Eng Prog 2005, 9:291-296.

20. Scheffner M, Romanczuk H, Münger K, Huibregtse JM, Mietz JA, Howley PM: Functions of human papillomavirus proteins. Curr Top Microbiol Immunol 1994, 186:83-99.

21. Hausen $\mathrm{H}$ : Papillomaviruses causing cancer: evasion from host-cell control in early events in carcinogenesis. J Natl Cancer Inst 2000, 92:690-698.

22. Luczak MW, Jagodzinski PP: Apicidin down-regulates human papillomavirus type $16 \mathrm{E} 6$ and E7 transcripts and proteins in $\mathrm{SiHa}$ cervical cancer cells. Cancer Lett 2008, 272:53-60.

23. Kenneth $J$, Thomas DS: Analysis of relative gene expression data using real-time quantitative PCR and the $2^{-\Delta \Delta C}$ method. Methods 2001, 25:402-408.

24. González MA: Molecular biology of cervical cancer. Clin Trans/ Oncol 2007, 9:347-354

25. Richardson $H$, Kelsall G, Tellier P, Voyer H, Abrahamowicz M, Ferenczy A, Coutlée F, Franco EL: The natural history of type-specific human papillomavirus infections in female university students. Cancer Epidemiol Biomarkers Prev 2003, 12:485-490.

26. Clifford GM, Rana RK, Franceschi S, Smith JS, Gough G, Pimenta JM: Human papillomavirus genotype distribution in low-grade cervical lesions: comparison by geographic region and with cervical cancer. Cancer Epidemiol Biomarkers Prev 2005, 14:1157-1164.

27. Vrese M, Rautenberg P, Laue C, Koopmans M, Herremans T, Schrezenmeir J: Probiotic bacteria stimulate virus-specific neutralizing antibodies following a booster polio vaccination. Eur J Nutr 2005, 44:406-413

28. Cross ML, Ganner A, Teilab D, Fray LM: Patterns of cytokine induction by gram-positive and gram-negative probiotic bacteria. FEMS Immunol Med Microbiol 2004, 42:173-180.
29. Pohjavuori E, Viljanen M, Korpela R, Kuitunen M, Tiittanen M, Vaarala O, Savilahti E: Lactobacillus GG effect in increasing INF-gamma production in infants with cow's milk allergy. J Allergy Clin Immunol 2004, 114:131-136.

30. Dugas B, Mercenier A, Lenoir-Wijnkoop I, Arnaud C, Dugas N, Postaire E: Immunity and probiotics. Immunol Today 1999, 20:387-390.

31. Jagtar S, Shweta S: Classification, regulatory acts and applications of nutraceuticals for health. Int J Pharm Biol Sci 2012, 2:177-187.

32. Romond MB, Haddou Z, Mie Icareck C, Romond C: Bifidobacteria and human health: regulatory effect of indigenous bifidobacteria on Escherichia coli intestinal colonization. Anaerobe 1997, 3:131-136.

33. Wang KY, Li SN, Liu CS, Perng DS, Su YC, Wu DC, Jan CM, Lai CH, Wang TN, Wang WM: Effects of ingesting Lactobacillus- and Bifidobacterium containing yogurt in subjects with colonized Helicobacter pylori. Am J Clin Nutr 2004, 80:737-741.

34. Silva AM, Barbosa FH, Duarte R, Vieira LQ, Arantes RM, Nicoli JR: Effect of Bifidobacterium longum ingestion on experimental in salmonellosis in mice. J Appl Microbiol 2004, 97:29-37.

35. Woodman CB, Collins S, Winter H, Bailey A, Ellis J, Prior P, Yates M, Rollason TP, Young LS: Natural history of cervical human papillomavirus infection in young women: a longitudinal cohort study. Lancet 2001, 357:1831-1836

36. Li GL, Jiang W, Xia Q, Chen SH, Ge XR, Gui SQ, Xu CJ: HPV E6 downregulation and apoptosis induction of human cervical cancer cells by a novel lipid-soluble extract (PE) from Pinellia pedatisecta Schott in vitro. J Ethnopharmacol 2010, 132:56-64.

Pre-publication history

The pre-publication history for this paper can be accessed here: http://www.biomedcentral.com/1741-7015/10/72/prepub

doi:10.1186/1741-7015-10-72

Cite this article as: Cha et al:: Antiviral activity of Bifidobacterium adolescentis SPM1005-A on human papillomavirus type 16. BMC Medicine 2012 10:72.

\section{Submit your next manuscript to BioMed Central and take full advantage of:}

- Convenient online submission

- Thorough peer review

- No space constraints or color figure charges

- Immediate publication on acceptance

- Inclusion in PubMed, CAS, Scopus and Google Scholar

- Research which is freely available for redistribution

Submit your manuscript at www.biomedcentral.com/submit
C Biomed Central 